\title{
AUTOMATED FORMOSAT IMAGE PROCESSING SYSTEM FOR RAPID RESPONSE TO INTERNATIONAL DISASTERS
}

\author{
M.C. Cheng ${ }^{\text {a }}$, S.C. Chou ${ }^{\text {a }}$, Y.C. Chen ${ }^{b}$, B. Chen ${ }^{a}$, C. Liu ${ }^{a}$, S.J. Yu ${ }^{a}$ \\ ${ }^{a}$ National Space Organization, 8F, 9 Prosperity 1st Road, HsinChu Science Park, HsinChu City, Taiwan \\ ${ }^{\mathrm{b}}$ GIS Research Center, Feng Chia University (GIS.FCU), No. 100, WenHwa Road, TaiChung City, Taiwan \\ Corresponding author: franz.cheng@nspo.narl.org.tw
}

ISPRS-IAA Space Agency Forum

KEY WORDS: FORMOSAT, Remote Sensing, Image Processing System, Disasters, Sentinel Asia, Nepal Earthquake

\begin{abstract}
:
FORMOSAT-2, Taiwan's first remote sensing satellite, was successfully launched in May of 2004 into the Sun-synchronous orbit at 891 kilometers of altitude. With the daily revisit feature, the 2-m panchromatic, 8-m multi-spectral resolution images captured have been used for researches and operations in various societal benefit areas. This paper details the orchestration of various tasks conducted in different institutions in Taiwan in the efforts responding to international disasters. The institutes involved including its space agency-National Space Organization (NSPO), Center for Satellite Remote Sensing Research of National Central University, GIS Center of Feng-Chia University, and the National Center for High-performance Computing. Since each institution has its own mandate, the coordinated tasks ranged from receiving emergency observation requests, scheduling and tasking of satellite operation, downlink to ground stations, images processing including data injection, ortho-rectification, to delivery of image products. With the lessons learned from working with international partners, the FORMOSAT Image Processing System has been extensively automated and streamlined with a goal to shorten the time between request and delivery in an efficient manner. The integrated team has developed an Application Interface to its system platform that provides functions of search in archive catalogue, request of data services, mission planning, inquiry of services status, and image download. This automated system enables timely image acquisition and substantially increases the value of data product. Example outcome of these efforts in recent response to support Sentinel Asia in Nepal Earthquake is demonstrated herein.
\end{abstract}

\section{INTRODUCTION}

FORMOSAT-2, Taiwan's first remote sensing satellite, was successfully launched in May of 2004 into the Sun-synchronous orbit at 891 kilometers of altitude. With the daily revisit feature, the 2-m panchromatic, 8-m multi-spectral resolution images captured have been used for researches and operations in the societal benefit areas of disaster, agriculture, forest, water, and many others. In the efforts to support responding to disasters, FORMOSAT-2 has been contributing to international initiatives or organizations such as Sentinel Asia, International Disaster Charter, United Nations Platform for Space-based Information for Disaster Management and Emergency Response (UNSPIDER), and UNITAR's Operational Satellite Applications Programme. Rapidness is the most critical factor for effectiveness in operations.

This paper details the orchestration of various tasks conducted in different institutes in Taiwan in the efforts responding to international disasters. The institutes involved including its space agency-National Space Organization (NSPO), Center for Satellite Remote Sensing Research of National Central University, GIS Center of Feng-Chia University, and the National Center for High-performance Computing (NCHC). The coordinated tasks ranged from receiving emergency observation requests, scheduling and tasking of satellite operation, downlink to ground stations, images processing include ortho-rectification, to delivery of image products. With the lessons learned from working with international partners, the FORMOSAT Image Processing
System has been extensively automated and streamlined with a goal to shorten the time between request and delivery in an efficient manner. The NSPO has developed an Application Interface for mobile access to its system platform that provides functions of search in archive catalogue, request of data services, mission planning, inquiry of services status, and image download. This automated system enables timely image acquisition and substantially increases the value of data product. Example outcome of these efforts in recent response to support Sentinel Asia in Nepal Earthquake is also demonstrated in this paper.

In 2016, the NSPO is preparing to launch its second remote sensing satellite, FORMOSAT-5, which will provide 2$\mathrm{m}$ panchromatic, 4-m multi-spectral resolution images. Considering the health conditions of FORMOSAT-2, joint operation of the two satellites is under planning. This automation effort in provision of high resolution image product chain will be shown to enhance the system operation performance and thus create even more benefits in rapid response to major disasters for international community.

\section{TAIWAN'S REMOTE SENSING SATELLITE MISSIONS}

\subsection{NSPO Space Missions}

Taiwan started her space technology development in 1991 by first establishing the National Space Program Office. It is later affiliated to the umbrella of the National Applied Research 
Laboratories, NARLabs, in 2003, and furthermore renamed to the National Space Organization in 2005. During the past 25 years, the development plan had been executed in 2 phases. Major achievements included erection of space infrastructure and facilities, development of indigenous space technology, and operation of multi-satellite and multi-mission. These achievements were demonstrated in the successful conduction of 3 space programs containing 8 Low Earth Orbit (LEO) satellites. In the First Phase Long Term Space Program, 19912004, 3 Earth observation satellite missions had been successfully conducted: FORMOSAT-1 (previously known as ROCSAT-1), a scientific satellite; FORMOSAT-2, a highresolution remote sensing satellite; and FORMOSAT3/COSMIC, a constellation of 6 meteorological micro-satellites. In this phase remote sensing and meteorological type of satellite applications were identified as the main focus that meets national needs. In the Second Phase, 2004-2018, Formosat-5 and Formosat-7 are being developed as follow-ons to FORMOSAT-2 and FORMOSAT-3, respectively. In this phase, emphasis is focused on developing indigenous capabilities in critical spacecraft component and instrument utilizing Taiwan's industrial strengths such as micro-electronics and others.

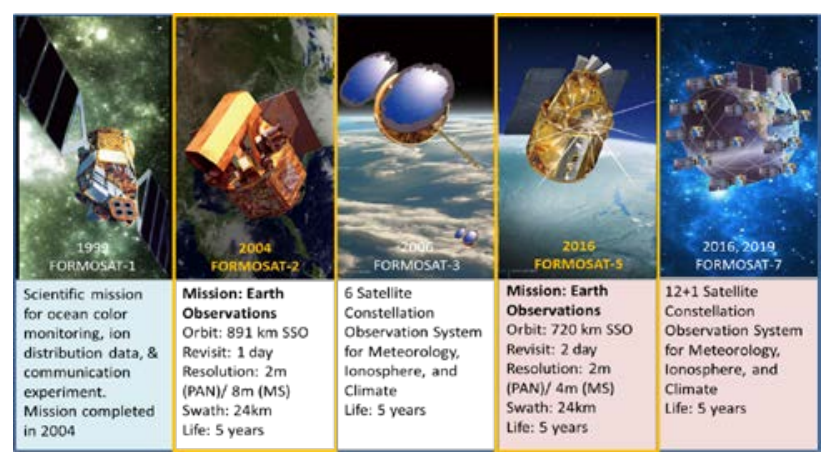

Figure 1. Satellite Missions of NSPO-NARLabs in Taiwan

\subsection{FORMOSAT-2 Mission}

FORMOSAT-2, Taiwan's first remote sensing satellite, was successfully launched in May of 2004 into the Sun-synchronous orbit at altitude of $891 \mathrm{~km}$. Major payload is a high-resolution electric-optical type Remote Sensing Instrument (RSI) with ground resolution of 2-m panchromatic (PAN, black \& white) and 8-m multi-spectral (MS, color). Images taken by FORMOSAT-2 not only fulfilled Taiwan civilian needs on land utilization, agricultural and forest planning, disaster assessments, and environmental monitoring, but they are also being distributed to international users for specific applications as shown in Figure 2.

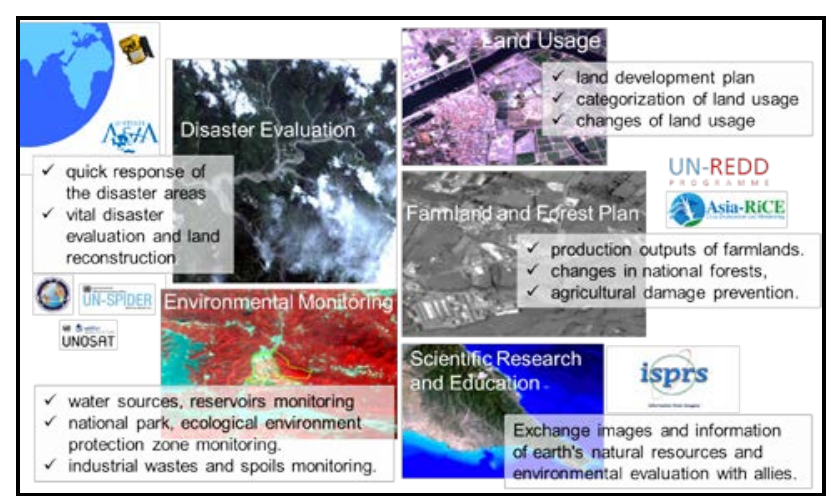

Figure 2. Applications of FORMOSAT-2 Images
Over the past decade, the footprint FORMOSAT-2, shown in Figure 3, has covered a surface area exceeding $1.2 \times 10^{9} \mathrm{~km}^{2}$, which is approximately 8 times the entire surface area of the world's land.

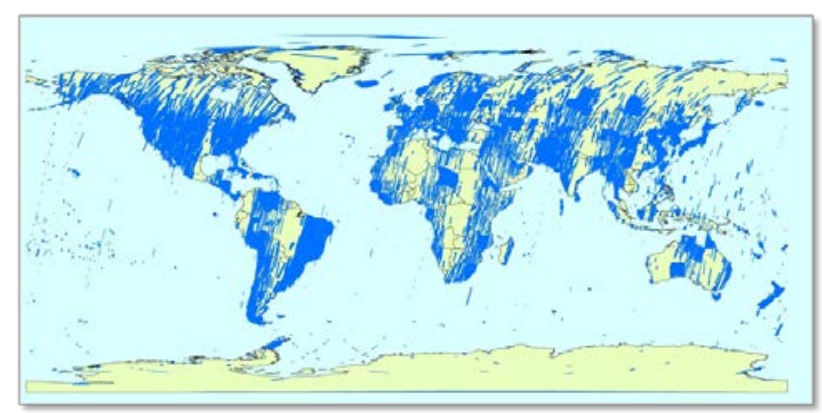

Figure 3. FORMOSAT-2 Footprint

The capacity of FORMOSAT-2 imaging coverage is shown in Figure 4. Due to the repetitive characteristic, the daily revisit region ranges $968 \mathrm{~km}$ around the ground track, which corresponds to the satellite roll angle of $45 \mathrm{deg}$ or the ground elevation angle of 36.3 deg. Some regions near the equator are not covered, but the total area is less than $18 \%$ of the global area. For emergency cases, the satellite can be operated at roll angles up to 53.6 deg to achieve the global coverage. ${ }^{[1]}$
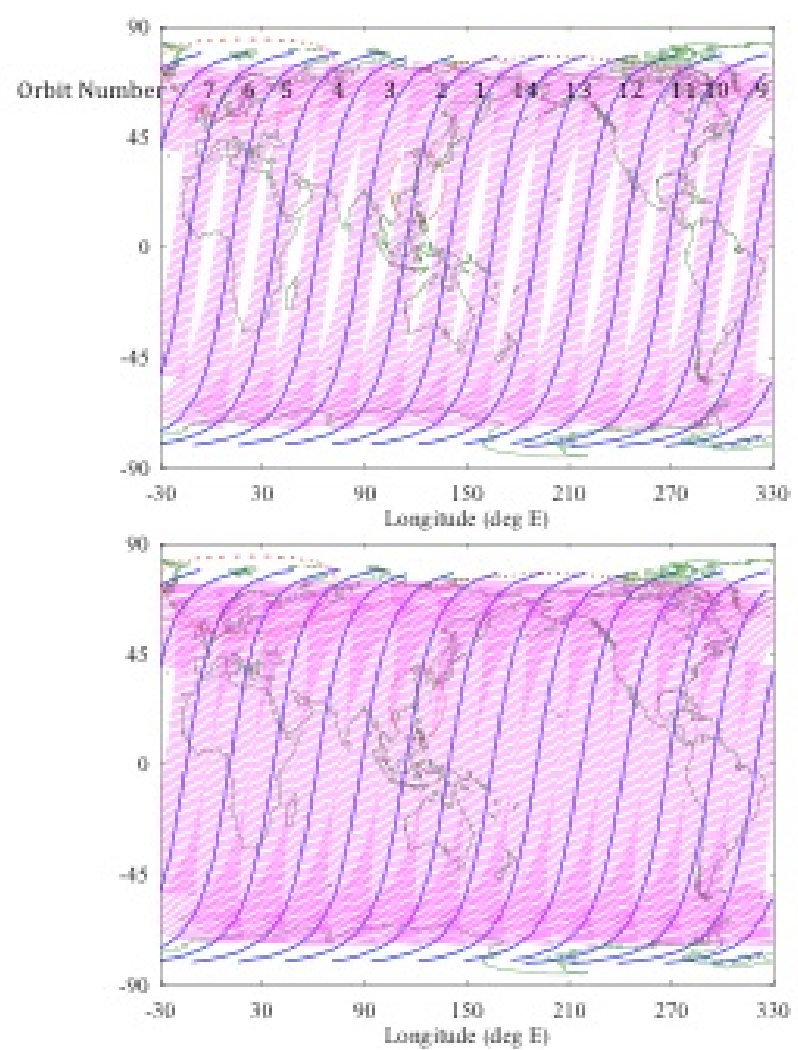

Figure 4. FORMOSAT-2 Ground Track with Field of Regard of 45 deg (upper) and 53.6 deg (lower).

\subsection{FORMOSAT-5 Mission}

Being a successor to FORMOSAT-2, FORMOSAT-5 is Taiwan's first indigenous Remote Sensing Satellite, which is scheduled to launch in June of 2016. It will operate in a Sunsynchronous orbit at $720 \mathrm{~km}$ of altitude and offers a temporal frequency of 2 days revisit. The optical RSI, the main payload, 
is equipped with an innovative five-band multi-SOC CMOS sensor that provides 2-m resolution panchromatic and 4-m resolution multi-spectral imagery. FORMOSAT-5 is capable of high agility manoeuvre, which enables asynchronous images tasking such as along the coastal line or along the longitude. Table 1 details the comparison of key satellite system parameters of FORMOSAT-2 and FORMOSAT-5 missions. FORMOSAT-5 is the first self-reliant remote sensing satellite designed and fabricated in Taiwan, it utilized domestic industrial strengths in IC foundry and chip implementation to support its space development.

Table 1. Key Parameters of FORMOSAT-2 vs. -5

\begin{tabular}{|c|c|c|}
\hline Key Parameter & FORMOSAT-2 & FORMOSAT-5 \\
\hline Orbit & SSO @ 891 km/99.10 & SSO @ 720 km/98.28 \\
\hline Revisit Period & 1 day & 2 days \\
\hline Mission Life & 5 years & 5 years \\
\hline GSD & PAN (2 m) / MS (8 m) & PAN (2 m) / MS (4 m) \\
\hline Swath & $24 \mathrm{~km}$ & $24 \mathrm{~km}$ \\
\hline Spectral Bands & $1 \mathrm{PAN}+4 \mathrm{MS}$ & $1 \mathrm{PAN}+4 \mathrm{MS}$ \\
\hline $\begin{array}{l}\text { RSI Image } \\
\text { Sensor }\end{array}$ & CCD & CMOS Image Sensor \\
\hline RSI duty Cycle & $8 \%$ & $8 \%$ \\
\hline Satellite Weight & $760 \mathrm{~kg}$ & $525 \mathrm{~kg}$ \\
\hline
\end{tabular}

As a contrast to Figure 4, Figure 5 shows the mission orbit of FORMOSAT-5, presenting a 2-day revisit period with a 45 deg normal field of regard.
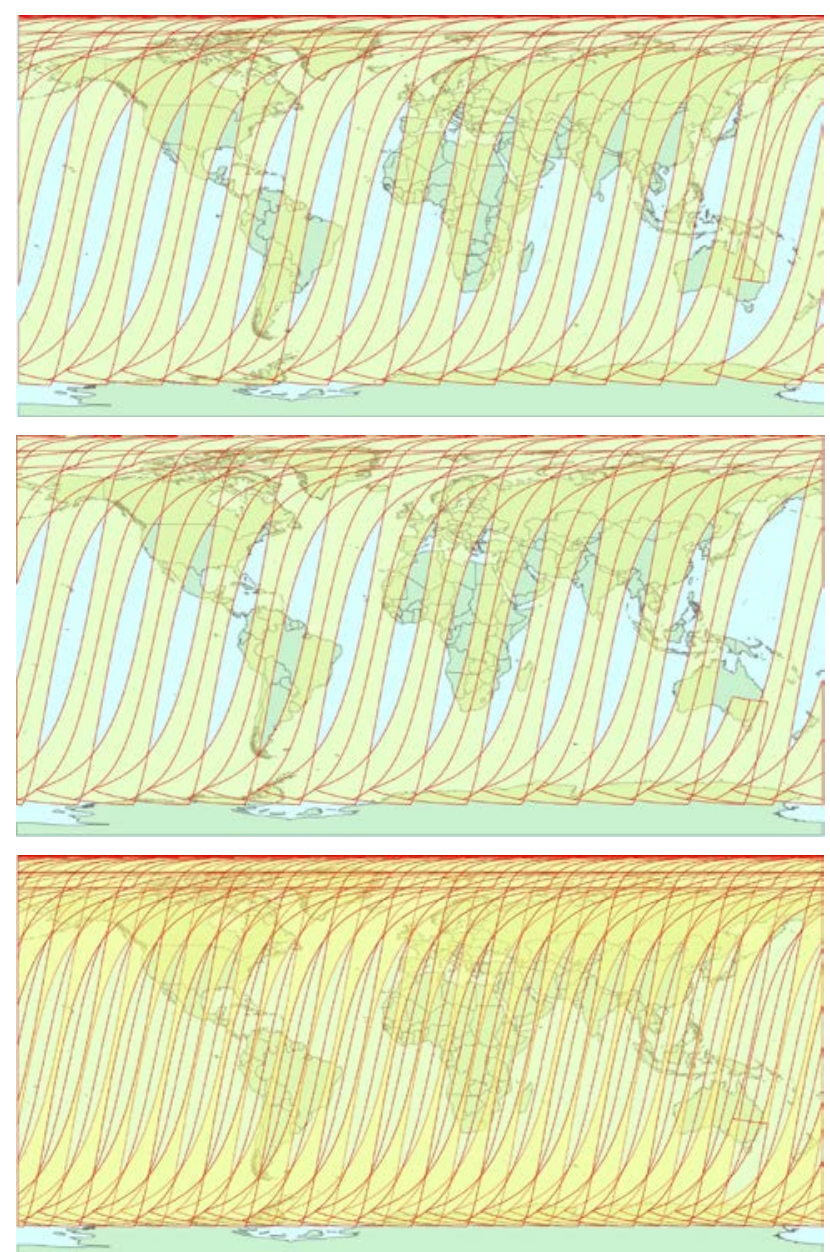

Figure 5. FORMOSAT-5 Ground Track with Field of Regard of 45 deg, Day 1 (upper), Day 2 (middle), Day 1+Day 2 (lower)
FORMOSAT-5 has completed the satellite system level Assembly-Integration-Testing activities in 2016 Q1. The launch campaign is targeted in 2016 as the major payload of SpaceX's Falcon 9 from Vandenberg, California in the United States. A performance simulation of the RSI has been conducted on the RSI Engineering Model, demonstrating that the image quality meets the requirement specifications. Figure 6 is an image taken from approximately $10 \mathrm{~km}$ south of the Taipei 101 building, which is $508.2 \mathrm{~m}$ high, a landmark of the dynamic Taipei City. Note that the image is before radiometric correction.

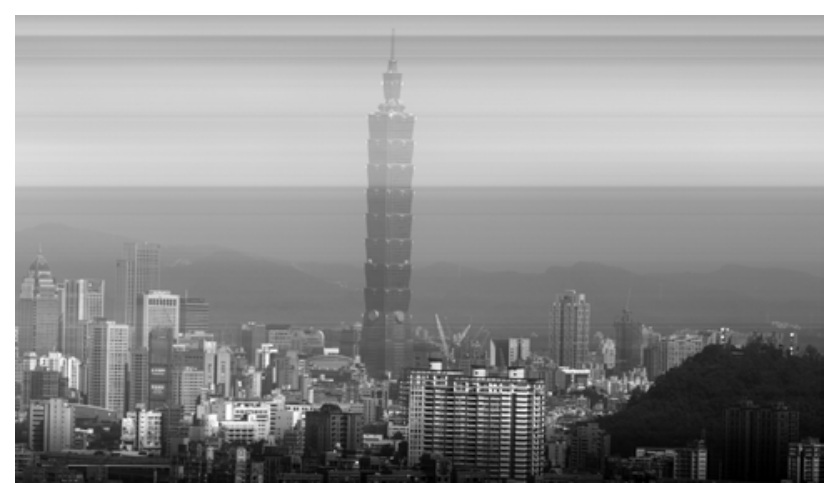

Figure 6. Remote Sensing Instrument Performance Simulation

\section{FORMOSAT-2 SUPPORTS TO INTERNATIONAL DISASTERS}

With its unique daily revisit capability and global coverage, FORMOSAT-2 is capable to get daily access to disaster areas and can provide near real time monitoring information for rescue planning. Since 2006, Taiwan has been providing free satellite images for humanitarian supports through international organizations including International Charter Space \& Major Disasters, UNOOSA/UN-SPIDER (United Nations Office for Outer Space Affairs/ Space-based Information for Disaster Management and Emergency Response), UNITAR/UNOSAT (United Nations Institute for Training and Research/ Operational Satellite Applications Programme) and Planet Action Initiative (since 2008). In 2010 FORMOSAT-2 joined the Sentinel Asia, and since then joined with other nation's or international organizations' efforts and contributed to fighting against natural disasters. The events supported include South Asia Tsunami (2004), Sichuan Earthquake (2008), Great East Japan Earthquake (2011), Haiyen typhoon (2014), and Nepal Earthquake (2015). Until 2015, FORMOSAT-2 has supported more than 57 countries in 236 events of major disaster reliefs.

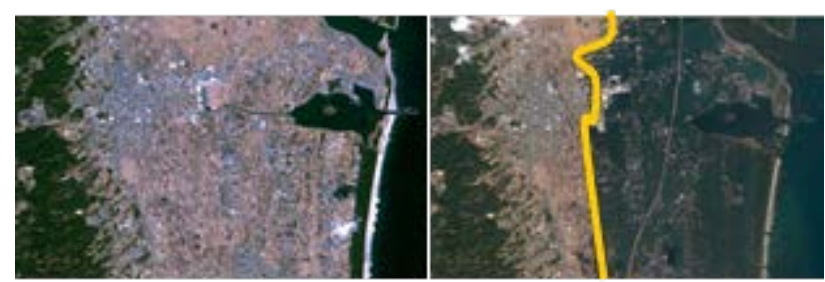

Figure 7. FORMOSAT-2 Images of Miyagi Prefecture Showing Tsunami Affected Area in Great East Japan Earthquake on 2011/3/11 (before disaster) vs. 3/12 (after)

Members in Sentinel Asia include Data Provider Node, Data Analysis Node, Disaster Management, and International Organizations such as Asian Development Bank. In response to major disasters, the Asian Disaster Reduction Center (ADRC) plays the roles in coordination of data/information provision 
and product end-users. In annual report, statistics of response time are discussed among the team members so that common problems could be identified. Emergency response time analysis results between June 2011 and October 2014 is summarized in Table $2^{[2]}$, which was reported in the 2015 Joint Project Team Meeting.

Table 2. Response Time Analysis Result under Sentinel Asia (Between June 2011 and October 2014)

\begin{tabular}{|l|c|c|c|c|c|}
\hline \multicolumn{1}{|c|}{$\begin{array}{c}\text { Days from } \\
\text { Request }\end{array}$} & Average & $\begin{array}{c}\text { Std. } \\
\text { Deviation }\end{array}$ & Min & Max & $\begin{array}{c}\text { No. of } \\
\text { Events }\end{array}$ \\
\hline To Activation & 0.53 & 0.94 & 0 & 6 & 70 \\
\hline $\begin{array}{l}\text { To Archive } \\
\text { Delivery }\end{array}$ & 2.26 & 1.95 & 0 & 9 & 50 \\
\hline $\begin{array}{l}\text { To Post Image } \\
\text { Delivery }\end{array}$ & 8.68 & 5.72 & 1 & 28 & 38 \\
\hline $\begin{array}{l}\text { To Product } \\
\text { Delivery }\end{array}$ & 20.89 & 10.44 & 7 & 41 & 28 \\
\hline
\end{tabular}

It is obvious that rooms for improvement for overall operation existed. It is thus recommended that all Sentinel Asia members worked together to a common goal in reducing the emergency response time. The NSPO has then reviewed internal standard operation procedures for effectiveness.

\section{AUTOMATION OF FORMOSAT IMAGE PROCESSING SYSTEM}

With growing usage of satellite and aerial photo in the internet application such as Google Earth, the image data itself becomes a commodity today. The performance of a remote sensing system is determined by its spatial, temporal, spectral and radiometric resolutions ${ }^{[3]}$ (Campbell, 2002). Therefore, the process to extract useful information from time-series data and the interpretation of human activity associated with each image become practical and important matter for application of emergency observation.

In the emergency period immediately after the occurrence of a disaster, it is difficult to obtain accurate and timely information from devastated area such as the exact location, the number of people affected and what exactly happened especially due to bad weather, demolished road and communication breakdown. However, during this critical stage, the fair-amount of information is crucial for a decision-maker to evaluate and manage the disaster. Therefore, a better temporal resolution satellite image together with high performance product generation system is highly desirable for the response and recovery in an emergency.

FORMOSAT-2 satellite is a satellite with daily revisit and global coverage features because it possesses capabilities of daily repeat, high altitude, and large field of regard. The multistripe imaging capability implemented by fully utilizing its manoeuvring agility enables contributing good benefits to an overall disaster assessment in its early stage. However, the large data volume can slow-down the data processing speed and increase the data latency. The problem was resolved after implementing a multi-processing-unit system and processing the imagery in parallel. Moreover, a semi-automatic speedy orthorectification system was also included in the processing chain to provide near real-time semi-ortho image product. The feature of large field of regard and high temporal frequency as well as innovative operation and performance of multi-processing-unit has been demonstrated by its rapid response for several emergency observations.

\subsection{Programming Capacity}

With the design of mission orbit, FORMOSAT-2 circulates the earth on sun-synchronous orbit of 14 revolutions per day. For each revolution, the satellite acquires images with duty cycle of $8 \%$, it orients the solar array to point to the Sun for the rest portion of daytime orbit and has orbit period of 102.9 minutes. Due to various kinds of disturbance, mission orbit shifts gradually but constantly. The programming system starts with a predicted orbit ephemeris and spacecraft attitude, and then produces a timeline and all of the parameters required to plan, schedule and command FORMOSAT-2. The optimal schedule for image tasking could be decided based on the constraints of attitude manoeuvre, orbital track, data storage and updated weather forecast. However, the characteristics of daily repeating orbit simplify operations, scheduling, and processing, and it is easy for the users to request images for urgent needs.

Meanwhile, on the strength of high torque and high momentum reaction wheels for control of spacecraft 3-axis inertial rates, FORMOSAT-2 is able to point up to 53.6 degree both along and cross track within 90 second. This agility allows the satellite to acquire large area in one single pass. Figure 8 shows that FORMOSAT-2 acquired Japan earthquake sensitive area by using two consecutive orbits. Although the image data suffers the degradation due to large viewing angle, it is beneficial to discriminate the changes of ground truth from the time-series images data because each image was taken under the similar illumination condition and geometric distortion. In general, the basic premise for change detection is that changes in the objects of interest will result in changes in reflectance values or local textures that are separable from changes caused by other factors such as differences in atmospheric conditions, illumination and viewing angles, and soil moistures. Under this approach, it is easy to remove the unrelated factor for change detection application.

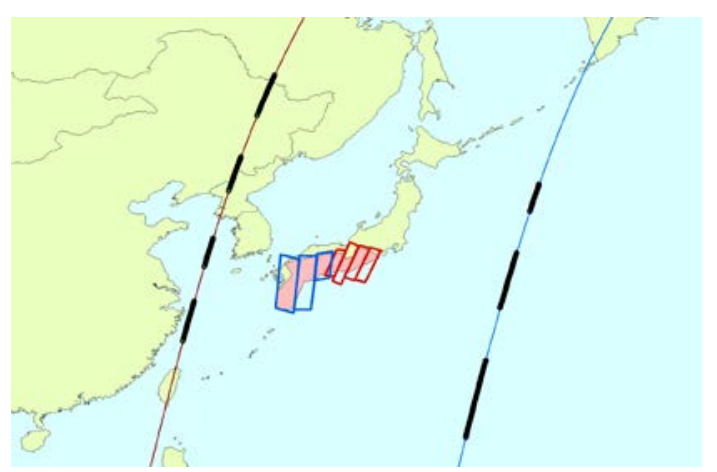

Figure 8. Illustration of FORMOSAT-2 Acquiring 7-Strips Images Data within 2 Hours by Using 2 Consecutive Orbits

\subsection{Scenario of FORMOSAT-2 Image Tasking}

In order to rapidly respond to emergency observation, the NSPO develops not only programming software system but also operation mechanism, which integrated satellite scheduling activity and mission analysis. Thanks for the characteristic of daily repeating orbit, it simplifies the operations, scheduling, and processing, and it is easy for the users to request images for urgent needs. $^{[1]}$ 
Due to orbit characteristics, there are two chances that ground station of Taiwan has the opportunity to contact with FORMOSAT-2 satellite. As shown in Figure 9, the satellite passes over Taiwan around Coordinated Universal Time, UTC 2 in the descending pass and around UTC 13 in the ascending pass. During this contact window, the data stored in the satellite storage will be downloaded to ground station and in the meanwhile scheduling commands will be uploaded to the satellite. The daily capacity of the scheduling volume is about 60 interested areas.

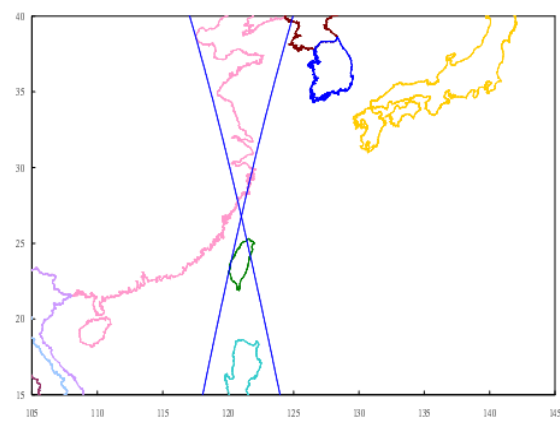

Figure 9. Ascending and Descending Pass over Taiwan.

In the event of the emergency, the NSPO will re-schedule the satellite activity and give higher priority to this urgent tasking. In general, according to the geo-location of disaster, the programming system will check the availably of satellite resource by taking following factors into account: satellite memory, the conflict of different tasking in the vicinity area of interest and orbit duty cycle limitation. The task of satellite programming is to optimize the usage of resource under those constraints.

The procedure timeline of emergency operation is shown in Figure 10. As long as the tasking request received before PM 4:00, the data will be downloaded on the next day to Taiwan. The data latency depends on the data volume. By means of the space infrastructure of oversea receiving station, it is expected that the time interval between commands upload and data download will be significantly shortened.

D-1

D

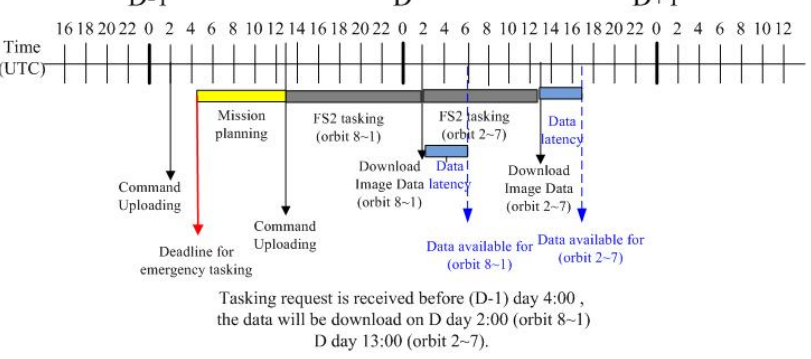

Figure 10. FORMOSAT-2 Schedule Timeline in Emergency Response Operation

\subsection{Fast Data Processing}

The NSPO develops FORMOSAT-2 Image Processing System (IPS) to generate image product. The IPS is designed to process images by scheduling image taking operations according to the user's needs. After the images are taken, the data will be downloaded through X-band antenna, processed by the IPS such as radiometric and geometric corrections, and then filed and stored in the computers. The IPS has been proven to be a reliable system for routine operation over the past decade. However, the raw data volume has grown far exceeding the original design specs in recent year due to the multi-stripe imaging need from the users. The large data volume can slowdown the data processing speed and sometimes cause system problem. As previously mentioned this issue was resolved by implementing a multi-processing-unit system and run the image processing simultaneously. The computers are connected in a network and each processor executes different image processing portions without interfering each other. On the other hand, the most challenging requirement is the end-to-end service delivery time at beginning of a crisis event. In order to provide first reference map, a speedy semi-automatic ortho-rectification system becomes necessary at this critical time. This system generates quasi-ortho-rectified products. This is necessary and can be considered as a compromise.

The architecture of this system, shown in Figure 11, is combination of Linux-based Image Processing System and hardware-accelerated ortho-rectification system. By using this multi-processing-unit product generation system, the NSPO can handle several segments at the same time for standard level $1 \mathrm{~A}$ and level 2 products generation. Meanwhile it takes approximately one minute to generate ortho-rectified product with geographic coordinates for panchromatic scene of size 12,000 multiplied by 12,000 . The geometric accuracy is up to 20 meters and depends on availability of ground control points. The overall throughput of system is estimated that the quasiortho-rectified products are available in 4 hours for an emergency event after the satellite downlink raw data is received from the $\mathrm{X}$-band antenna.

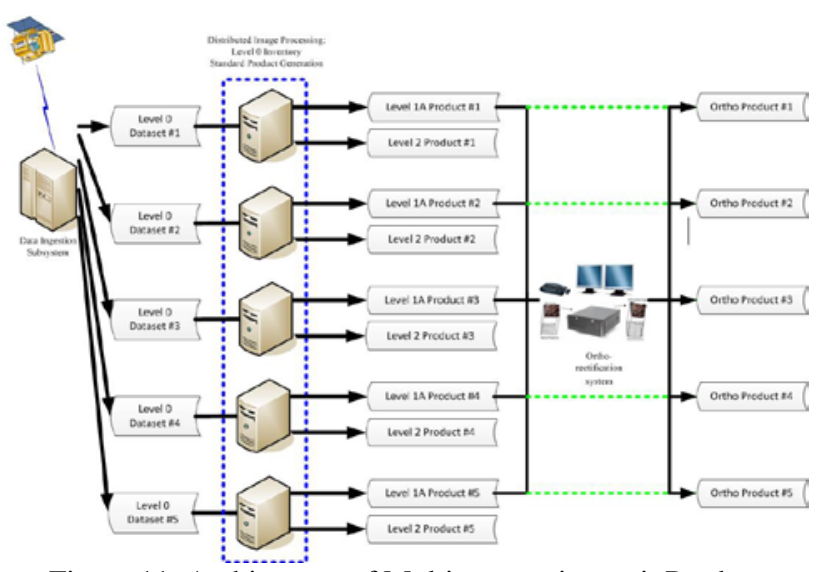

Figure 11. Architecture of Multi-processing-unit Product Generation System ${ }^{[4]}$

\subsection{FORMOSAT-2 Operating Mechanism in Emergency}

To enhance the data processing of the emergency events means each stage of data processing procedures can be more easily and reduce the manual work time in a systematic way to ensure products successfully submitted. Figure 12 describes the framework for the Data Processing of the Emergency Events. A Catalogue Query API has been designed to connect tasking request, and the backend server obtains the relevant information to transfer from each stage and the framework diagram refer to Figure 12. Note that the CSRSR operates FTP Data Sync Setting Program, and Web Query System provides the complete WMS (Web Map Service) publishing path from the NCHC, as well as the file condition of the operation process. 


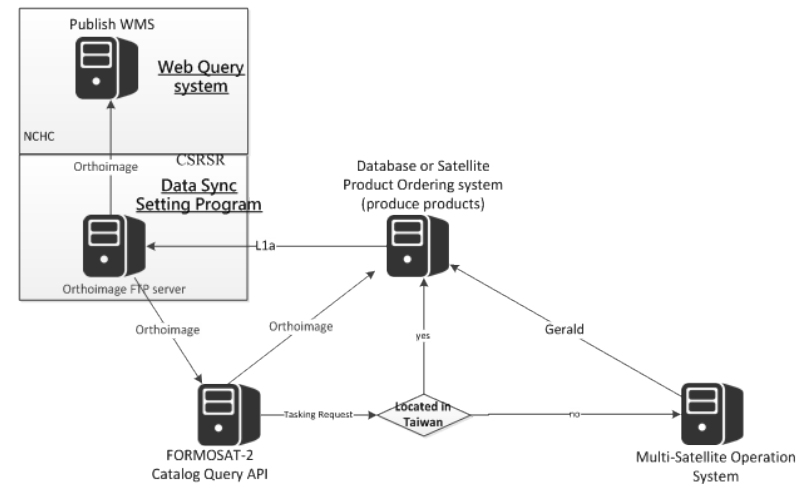

Figure 12. Framework for the Data Processing of the Emergency Events

\subsection{Data Sync Setting Program (GUI Program Installed on FTP Server)}

When the product is completed, the product is synchronized to Satellite Product Ordering System through scheduling services. The product is packaged automatically and synchronized to FTP server at the CSRSR so that the relevant members can receive notifications that the product has been completed, as shown in Figure 13. Note that the automated production system replaces manual work and then supplies products to the CSRSR.

FTP data at the CSRSR is set up synchronously as a GUI (Graphical User Interface) program and installed on FTP server of the CSRSR. The purpose is to provide the CSRSR complete ortho-images and to synchronize to the NSPO and the NCHC as a following source. During the emergency image-taking tasks, when high cloud ratio images or incomplete ortho-images are acquired, this program will provide as-is inputs and synchronize to the NSPO and the NCHC.

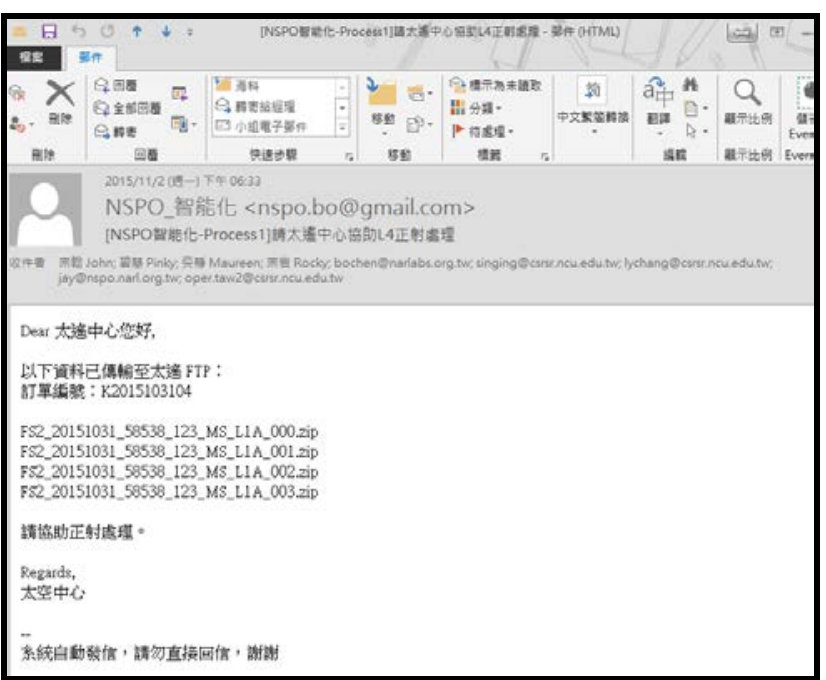

Figure 13. Notification that NSPO Product Sync to CSRSR

\subsection{Web Query System}

Web Query System is installed on Satellite Database Quick Search and Real Time Service System in the NCHC. When the NCHC receives the notification of ortho-image, WMS can be released. Web Query System enters the link such as Figure 14, and notifies the relevant staff afterwards as shown in Figure 15.

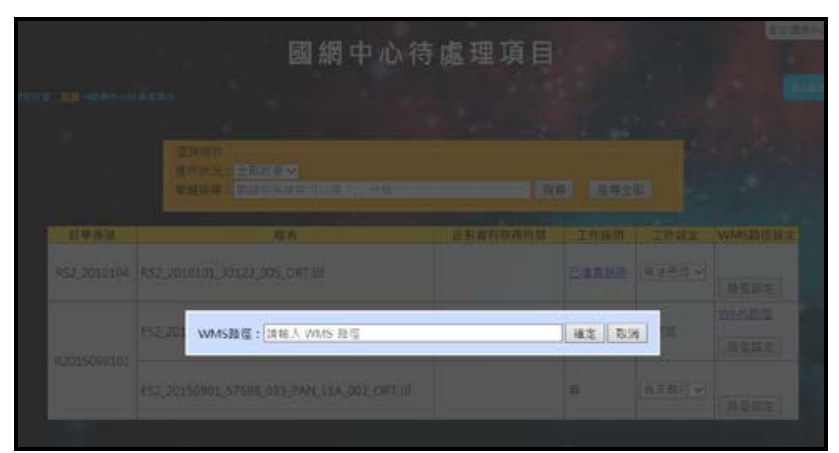

Figure 14. WMS Path

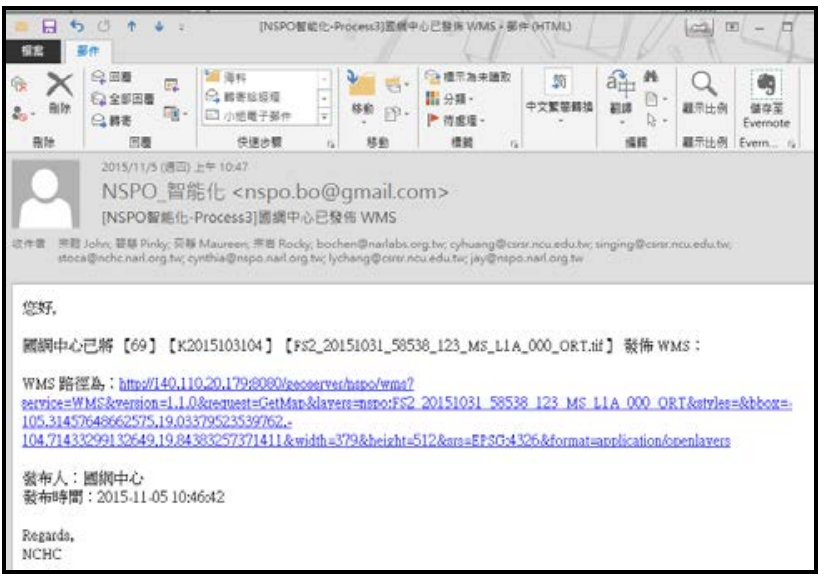

Figure 15. Notification of WMS Implementation

In addition, relevant members can track the file status of each order processing as shown in Figure 16.

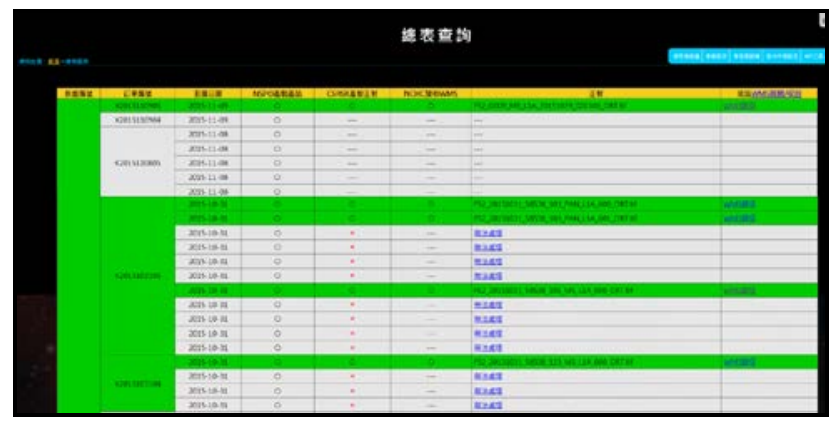

Figure 16. Processing Status of Each Order

\section{EXAMPLE IN NEPAL EARTHQUAKE}

In The 2015 Nepal earthquake, this team also provided WMS information of post-disaster images and completed by NSPO, CSRSR, GIS.FCU, and NCHC. The 2015 Nepal earthquake struck Nepal at UTC 06:11 on 25 April with a magnitude of 7.8 and at a depth of approximately $15 \mathrm{~km}$, followed by aftershocks including a large magnitude 7.3 quake on 12 May. The initial earthquake struck with an epicenter in the mountains to the northwest of Kathmandu, and aftershocks around the city of Kathmandu. The data reported by the United States Geological Survey (USGS). This earthquake killed more than 8,000 people and injured more than 21,000. The NSPO received the tasking request around UTC 08:00 and started to program the FORMOSAT-2 satellite to acquire image over Kathmandu area. The satellite command was uploaded at UTC 13:20 on 25 April when FORMOSAT-2 passed over Taiwan. The first image was 
taken at UTC 03:46 on 26 April. It is approximately 21 hours just after the earthquake occurrence.

Table 3. FORMOSAT-2 Response for Nepal Earthquake

\begin{tabular}{|l|r|r|r|r|}
\hline \multicolumn{1}{|c|}{$\begin{array}{c}\text { Target } \\
\text { Name }\end{array}$} & cent_lat & cent_lon & $\begin{array}{c}\text { pitch } \\
\text { angle, }\end{array}$ & roll angle \\
\hline Kathmandu & 27.7313 & 85.3464 & -9.0514 & -46.5633 \\
\hline Mt. Everest & 27.9242 & 86.8296 & 24.9312 & -41.3096 \\
\hline Dhading & 27.9833 & 84.6804 & -44.2160 & -35.4362 \\
\hline Kodari & 28.5619 & 86.1488 & -0.2896 & -45.7685 \\
\hline $\begin{array}{l}\text { Earthquake_ } \\
\text { Dolakha }\end{array}$ & 28.3083 & 86.0126 & 3.5409 & -46.0044 \\
\hline
\end{tabular}

During the emergency, accurate data and comprehensive information will help the decision makers make better decision. Therefore, in addition to semi-automatic processing of quasiortho-rectified processing, the NSPO also collaborates with the CSRSR of NCU and the NCHC to produce level-4 image, which has higher geometrical accuracy. The collaboration framework is shown in Figure 17. This mechanism adopts OGC protocol to distribute ortho-image and GIS capacity via WMS, as shown in Figures 18 and 19.

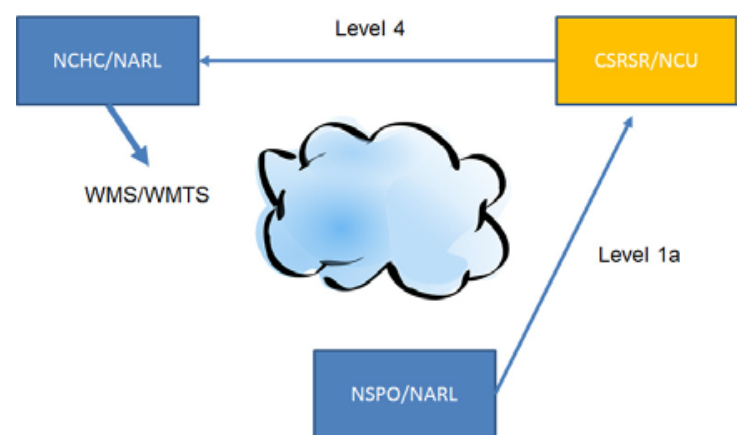

Figure 17. NSPO-CSRSR-NCHC Collaboration Framework

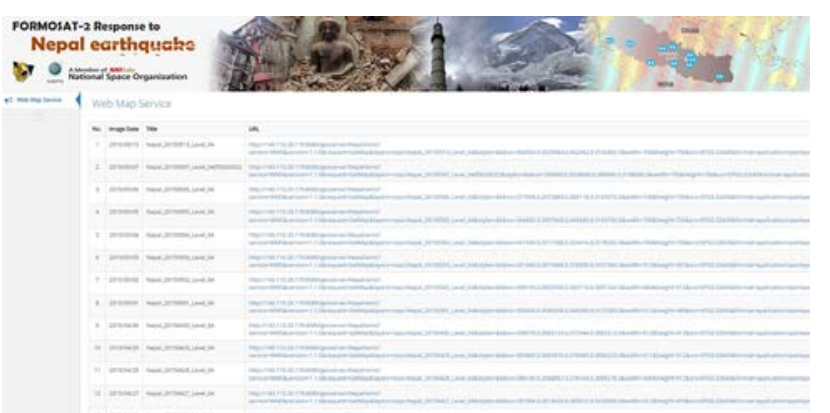

Figure 18. Web Map Service of FORMOSAT-2 Image for Supporting in Nepal Earthquake
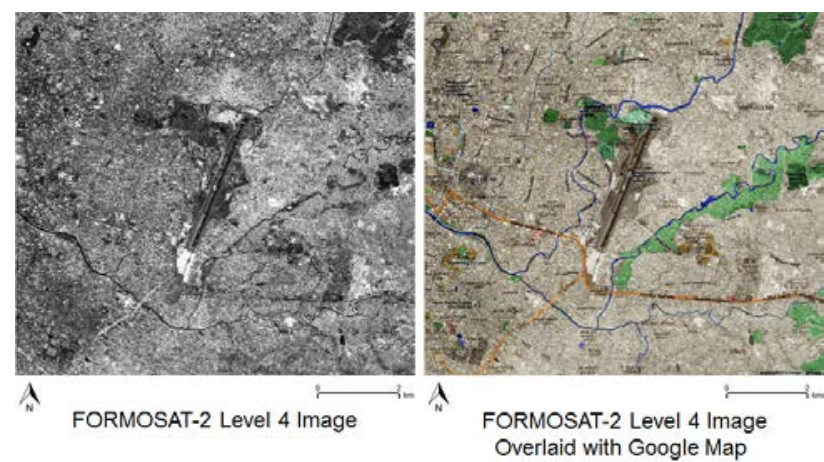

Figure 19. FORMOSAT-2 Level 4 Image of Kathmandu, Nepal

\section{SUMMARY}

\subsection{Concluding Remarks}

Taiwan is frequently struck by natural disasters such as typhoon, flood, earthquake, landslides and others. As a small country with limited resources, its space agency-the NSPO developed Earth observations satellite missions to monitor the nation's environments. The FORMOSAT-2 images have been used and contributed to disaster responses. In order to make the operation efficient and effective, a value-chain to produce satellite image is coordinated by synergizing the NSPO IPS and other domestic institutions-CSRSR of NCU, the GIS Center of FCU, and the NCHC. The value-chain with coordinated tasks ranged from receiving emergency observation requests, scheduling and tasking of satellite operation, downlink to ground stations, images processing including data injection, ortho-rectification, to delivery of image products to the users. The achievements supporting the domestic operations had been extended to international community such as International Disaster Charter, UNSPIDER, UNOSAT, and especially Sentinel Asia.

With the lessons learned from working with international partners, in recent years, the FORMOSAT-2 IPS has been extensively automated and streamlined with a goal to shorten the time between request and delivery in an efficient manner. The integrated team has developed an Application Interface for mobile access to its system platform that provides functions of search in archive catalogue, request of data services, mission planning, inquiry of services status, and image download. This automated system enables timely image acquisition and substantially increases the value of image product. Example outcome of these efforts in support to Sentinel Asia in April 2015 Nepal Earthquake is demonstrated herein.

\subsection{Future Work}

The integrated NSPO-CSRSR-GIS.FCU-NCHC team has synergised each institution's core competences and established related mechanisms into a value chain for an emergency response task and increased the effectiveness to achieve greater societal benefits. Future recommendations and improvements are as follows. However, there is also room for improvement. Foe example, the CSRSR currently produces ortho-images with segment, which is not corresponding to the satellite planning grid system. The process program could be further revised to generate grid-based level-1A data in a form of ortho-rectified image. It is observed that the Web Query System is to be established so that related scheduling service connects to the NSPO satellite programming system. The work has ability to anticipate reference map production when a sign of crisis occurred. It is expected to further shorten the "latency time" and to reduce the delivery delay with respect to the effective user request.

Currently, the NSPO has completed developing the space segment of FORMOSAT-5, which is a follow-on mission to FORMOSAT-2. The launch campaign is scheduled in August 2016. With this experience in IPS and continuing improvements, the NSPO is committed to providing better quality rapid response services to the international community in the joint efforts fighting against natural disasters. 


\section{REFERENCES}

[1] An-Ming Wu, Guey-Shin Chang. Quick Response for Disaster Monitoring from FORMOSAT-2 Satellite. IAC-10B1.1.6, 61 ${ }^{\text {st }}$ International Astronautical Congress (IAC 2010), Prague, Czech, September 27-October 1, 2010.

[2] Ming-Chih Cheng, Kazuya Kaku, Yu-Ching Liu, Takehisa Chiba, Bo Chen, Shiann-Jeng Yu, Guey-Shin Chang. Taiwan’s FORMOSAT Satellites in Responses to Asia Pacific Regional Disasters with Sentinel Asia. IAC-15-B1.1.6, 66 ${ }^{\text {th }}$ International Astronautical Congress (IAC 2015); Jerusalem, Israel; October 12-16, 2015.

[3] J. B. Campbell, Introduction to Remote Sensing, 3rd ed. London, U.K.: Taylor \& Francis, 2002.

[4] Bo Chen, Shih-Chieh Chou, L.H.Chang. The FORMOSAT2 Rapid Response for Emergency Observation. 32 ${ }^{\text {nd }}$ Asian Conference on Remote Sensing (ACRS 2011); Taipei Taiwan; October 3-7, 2011. 